COMO A DESIDRATAÇÃO PODE AFETAR A PERFORMANCE DOS ATLETAS

OLGUIN, Larissa Beatriz Pessoa ${ }^{1}$
BEZERRA, Angélica Cristiane Bernardes ${ }^{2}$
SANTOS, Vanessa Paes dos ${ }^{2}$

\begin{abstract}
RESUMO: A pesquisa aborda através de uma revisão bibliográfica, o conhecimento da hidratação na prática esportiva. A ingestão hídrica é um componente de suma importância na preparação do atleta profissional, amadores e praticantes de atividade física, porém, nem sempre recebem os cuidados que necessitam, um erro poderá implicar em consequências graves no rendimento e também na saúde em geral. Devido à necessidade da manutenção do estado de hidratação, torna-se necessária sua avaliação antes, durante e depois dos exercícios físicos, a perda hídrica através da sudorese, especialmente quando é realizado em um ambiente de temperaturas elevadas. Tal perda pode acarretar desidratação, alteração do equilíbrio hidroeletrolítico, dificuldade de efetuar a termorregulação, e desse modo, pode causar uma queda do desempenho esportivo. Diante disso, a reposição de líquidos é essencial para a manutenção do desempenho, e principalmente para garantir um funcionamento satisfatório do sistema cardiovascular. Neste contexto, a seleção de uma bebida esportiva para ser ingerida antes, durante e após exercícios com objetivo de reposição dos fluidos corporais perdido pode ser interessante, e deve respeitar algumas características específicas para promover uma recuperação hidroeletrolítica e metabólica adequada. Conclui-se, propor orientações sobre a hidratação em atletas e praticantes de atividade física, informando sobre a importância da ingestão hídrica para que não sejam prejudicados o desempenho esportivo e a saúde como um todo.
\end{abstract}

Palavras-chave: Hidratação. Desidratação. Esporte. Atleta. Performance.

\title{
HOW DEHYDRATION CAN AFFECT THE PERFORMANCE OF ATHLETES
}

\begin{abstract}
SUMMARY: The research approaches through a bibliographical review, the knowledge of hydration in sports practice. Water intake is a very important component in the preparation of the professional athlete, amateurs and practitioners of physical activity, however, they do not always receive the care they need, a mistake can imply serious consequences on income and also on health in general. Due to the need to maintain the hydration state, it is necessary to evaluate the water loss through sweating before, during and after exercise, especially when it is performed in a high temperature environment. Such loss may lead to dehydration, altered hydroelectrolyte balance, difficulty in effecting thermoregulation, and thus may cause a drop in athletic performance. Therefore, the replacement of liquids is essential for maintaining performance, and especially to ensure a satisfactory functioning of the cardiovascular system. In this context, the selection of a sports drink to be ingested before, during and after exercises aimed at restoring lost body fluids may be interesting, and must respect some specific characteristics to promote adequate hydroelectrolytic and metabolic recovery. It is concluded, propose guidelines on hydration in athletes and practitioners of physical activity, informing about the importance of water intake so that sports performance and health as a whole are not impaired.
\end{abstract}

Keywords: Hydration. Dehydration. Sport. Athlete. Performance.

\section{INTRODUÇÃO}

A água é a molécula mais abundante do organismo e também e consumida em maior quantidade

\footnotetext{
${ }^{1}$ Nutricionista, Pós-Graduada em Nutrição Esportiva e Nutrição Clínica Funcional e Fitoterápica pela Faculdade Laboro, Brasília/DF

${ }^{2}$ Nutricionista, Pós-Graduada em Nutrição Esportiva, pela Faculdade Laboro, Brasília/DF
} 
que qualquer outro nutriente. A necessidade diária de água varia individualmente sendo influencia por uma série de fatores, como as condições ambientais, características das atividades físicas, duração da sessão, intensidade do exercício e necessidade de vestimentas que interferem na termorregulação (RIBEIRO; LIBERALI, 2010).

Segundo Cirne et al (2011), muitos atletas profissionais, amadores e praticantes de atividade física, não realizam adequada ingestão hídrica diária que deve ser feita com objetivo de repor o que foi perdido durante a prática esportiva. Estes habitualmente negligenciam ou desconsideram a importância dessa conduta para o seu desempenho. Em decorrência disto, estima-se que muitos iniciam treinamento e provas de competição em estado de desidratação que é considerado a transição entre o estado de hidratação para hipohidratação.

O consumo da hidratação é essencial antes, durante e após uma atividade física ou competição. Diante dessas situações pode surgir a desidratação, com o desempenho aeróbio reduzido, o que vem acarretar uma redução no volume sanguíneo e aumento da frequência cardíaca, em atividades leves a moderadas, pode manifestar, fadiga, perda de apetite, sede, pele vermelha, tontura, oligúria e aumento da concentração urinária, quando graves ocorre dificuldade deglutição, perda de equilíbrio, a pele se apresenta seca e murcha, olhos afundados e visão fosca, disúria, pele dormente, delírio e espasmos musculares (NOBREGA et al, 2009).

É favorável que se consuma líquidos antes de sentirem sede, pois um dos sinais e sintomas da desidratação, quando leve a moderada, é a sede, apontando que já existe uma desidratação mínima de $1 \%$ a $2 \%$, o que já seria suficiente para reduzir a performance. Nesse contexto, o consumo inadequado de liquido pode ocasionar a desidratação, em altos índices pode surgir consequências como colapso respiratório, choque hipertermico e até mesmo à morte do atleta (PEREIRA et al, 2010).

A seleção da bebida para ser consumida no contexto esportivo precisa considerar as características da modalidade para promover uma recuperação necessária. Com isso, a formulação de bebidas destinadas a hidratação deve ser direcionada a especificidade do exercício, apesar de não existir uma bebida que contemple integralmente a necessidade de cada desporto. As recomendações em relação às características de uma bebida ideal destinada à hidratação e o quanto consumir nos momentos antes, durante e após exercício (HAUSEN et al, 2013).

Portanto, o objetivo do presente estudo é verificar por meio de uma revisão de literatura a importância de fornecer informações sobre a hidratação, desidratação e o consumo de algum tipo de bebida específica, antes, durante e após a prática esportiva.

\section{A IMPORTÂNCIA DA HIDRATAÇÃO NA ATIVIDADE FÍSICA}

A água é o elemento mais encontrado no corpo humano que constitui de 45 a $60 \%$ do peso corporal, sendo um nível adequado no corpo é essencial para sobrevivência, a prática de exercício prolongado, principalmente em ambientes quentes, resulta na perda significativa de água, potencializando o surgimento de estresses fisiológicos relacionadas ao calor, como, por exemplo: a desidratação e consequente hipertermia. Portanto, um atleta submetido a um treinamento intenso com uma alta temperatura ambiental pode perder vários litros de suor em um único dia, podendo chegar ao extremo de 10 a 12 litros, o que equivale a cerca de $25 \%$ do conteúdo de água de um homem de porte médio (PEREIRA et al, 2010).

A importância da reposição de líquidos durante a atividade física é comprovada por muitos estudos. Além do tipo de bebida, a caracterização das práticas e a avaliação do estado de hidratação 
constituem elementos importantes para garantir uma hidratação adequada. Devido à necessidade do estado de hidratação do indivíduo, torna-se necessária à sua avaliação antes, durante e depois dos exercícios físicos, principalmente quando realizados de longa duração e em condições climáticas desfavoráveis à termorregulação (BOURA; LIMA, 2011).

Alguns procedimentos requerem o conhecimento de fatores que influenciam de quando e quanto beber de água, estes são fatores que dependem da atividade, condicionamento físico, idade, estresse ambiental, e principalmente da individualidade do atleta. Efeitos da desidratação podem ocorrer seja esta, leve ou moderada com até $2 \%$ de perda da massa corporal, agravando-se à medida que ela se acentua (SILVA et al, 2015).

O descuido no consumo de líquidos, por atletas que se exercitam em locais quentes e úmidos, comuns em provas, podem provocar danos fisiológicos e neuropsicológicos, desfavorecendo o desempenho cognitivo-motor. Decréscimos no desempenho cognitivo-motor podem ocorrer quando $2 \%$ ou mais é perdida devido à restrição de líquidos, calor e/ou esforço físico. Embora uma redução de $2 \%$ a 3 $\%$ reduz o desempenho cognitivo-motor, que perde de apenas $1 \%$ já seria o suficiente para tal efeito. Visto que de acordo com Barroso et al (2014):

Dependendo do aspecto, o prejuízo físico e cognitivo-motor podem ser aparentes depois de uma perda de entre $1 \%$ a $10 \%$. Porém, estudos com efeitos do estado de hidratação na função cognitiva-motora ainda permanecem obscuros e carecem de maior investigação.

Os efeitos fisiológicos da desidratação induzida pelo exercício em estudos, através da comparação de diversas respostas fisiológicas de indivíduos que não repõem as perdas de líquido durante um exercício prolongado, ou as repõem parcial ou totalmente. Neste sentido, uma atenção especial deve ser dada a temática hidratação devido a sua influência direta no rendimento (RIBEIRO; LIBERALI, 2010).

A perda hídrica pela sudorese durante o exercício também pode levar o organismo à desidratação, com aumento da osmolaridade, da concentração de sódio no plasma e diminuição do volume plasmático. Quanto maior a desidratação, menor a capacidade de redistribuição do fluxo sanguíneo para a periferia, menor a sensibilidade hipotalâmica para a sudorese e menor a capacidade aeróbica para um dado débito cardíaco (JAHNKE, 2011).

Para garantir que o indivíduo inicie a prática esportiva no estado de hidratação, recomenda-se a ingestão de cerca de 250 a $500 \mathrm{ml}$ de água duas horas antes do exercício. Durante atividade física recomenda-se iniciar a ingestão hídrica nos 15 minutos e continuar bebendo a cada 15 a 20 minutos. Se o exercício tiver duração de mais de uma hora, ou se for intensa do tipo intermitente mesmo com menos de uma hora, deve-se repor carboidratos na quantidade de 30 a $60 \mathrm{~g}$ e sódio na quantidade de 0,5 a $0,7 \mathrm{~g}$. A temperatura ideal para a bebida deve ser em torno de 15 a $22^{\circ} \mathrm{C}$ e conter uma preferencial do indivíduo. Após o exercício, deve-se continuar ingerindo líquidos para compensar as perdas adicionais de água pela urina e sudorese (CIRNE; MENDES, 2011).

\subsection{Consequências da Desidratação}

A desidratação é uma condição fisiológica decorrente de uma prolongada perda hídrica corporal. Podendo afetar as funções fisiológicas e a temperatura corporal, desencadeando complicações e prejudicando o desempenho durante o exercício (WEBBER et al, 2009).

A desidratação pode causa redução da volemia, tornando o atleta mais suscetível à hipotensão postural e colapso. A baixa volemia também está associada à redução do volume de ejeção cardíaco que resulta na redução do fluxo sanguíneo para a pele, com efeito negativo na dissipação do calor. Quanto 
maior a desidratação, menor a capacidade de redistribuição do fluxo sanguíneo para a periferia, menor sensibilidade hipotalâmica para a sudorese e menor capacidade aeróbica para um dado débito cardíaco. A temperatura retal em atletas desidratados pode se apresentar maior que a de atletas em estado de hidratação normal (RIBEIRO; LIBERALI, 2010).

Os efeitos fisiológicos da desidratação induzida pelo exercício têm sido estudados através da comparação de diversas respostas fisiológicas de indivíduos quando estes não repõem as perdas de líquido durante um exercício prolongado, ou as repõem parcial ou totalmente. Há uma diminuição no volume plasmático com o início do exercício. Esta redução é influenciada pelo tipo e pela intensidade do exercício, assim como pela postura adotada. Em consequência, há uma redução progressiva do volume plasmático associada ao exercício, que pode ser compensada pela ingestão de líquidos durante o mesmo (MOREIRA et al, 2006).

Ao realizar um exercício com a duração superior há uma hora, o principal fator para a queda da performance é a depleção de glicogênio muscular e principalmente os problemas relacionados com a termorregulação e balanço hídrico. Quando a temperatura e a umidade do ambiente estão altas, a capacidade de manter a atividade física é reduzida; nesta situação, o processo de desidratação e sua influência sobre os mecanismos de termorregulação é um importante fator determinante da fadiga. Para Lima et al (2007):

A elevação da temperatura corporal pode prejudicar as respostas fisiológicas e o desempenho físico produzindo risco à saúde. Essa perda ocorre principalmente pelo suor, que é a mais importante forma de dissipar calor. A produção de suor pode levar a hipohidratação, ocorrendo diminuição da performance, comprometimento do sistema cardiovascular e uma demanda do suprimento de sangue para a pele e músculo além de ocorrer alterações na função renal e nos hormônios associados com a retenção de fluído e sódio.

Para tanto, é indicado que os atletas iniciem o exercício bem hidratados, sendo que o estado de hiper-hidratação protege contra o estresse térmico, retardando a desidratação, aumenta a transpiração durante o exercício e minimiza a elevação da temperatura central, melhorando então o desempenho (WEBBER et al, 2009).

Segundo Pinto et al (2014), o treinador tem por objetivo levar seus atletas a um desempenho elevado. O controle fisiológico do corpo durante o exercício físico evita o esgotamento muscular, controla o glicogênio hepático e previne a desidratação. O estado de hidratação é um fator determinante para a prática de atividade física. Atualmente existem diversos métodos de avaliação da hidratação utilizados. São eles: marcadores simples (coloração urinária e massa corporal), marcadores complexos (água corporal total e osmolaridade plasmática), análises em amostras sanguíneas, entre outros marcadores (concentração de hemoglobina e hematócrito, sódio plasmático e sede).

Estudos realizados com competidores de mountain bike devido a desidratação foram observadas algumas manifestações como: sensação de perda de força, câimbras, sede muito intensa, fadiga generalizada, dor de cabeça, dificuldade de realização de um movimento técnico facilmente realizado em condições normais, perda de sensibilidade nas mãos, dificuldade de concentração, e ainda outros sintomas com menor número de manifestações entre os atletas. Entre as soluções mais consumidas como agente reidratante, tem-se a água, isotônico, além do suco natural e refresco. O consumo de bebidas gasosas durante o exercício causa desconforto gástrico, e o consumo de bebidas alcoólicas resulta em desidratação, o que prejudica o desempenho do atleta (CRUZ et al., 2009).

Em outros estudos com nadadores adolescentes, um dos principais sintomas de desidratação é a sede, no entanto neste esporte, o nadador pode ter essa percepção reduzida, pois estará com a boca em 
contato com a agua durante todo o exercício estimulando os receptores nervosos localizadas na região orofaríngea, dando a sensação como estivesse hidratando-se constantemente. A completa recuperação pósexercício envolve alguns processos fisiológicos como: retorno à normalidade das funções fisiológicas, diminuição das dores musculares, desaparecimento dos sintomas fisiológicos associados a fadiga e rearmazenamento de fluídos e reservas energéticas. A bebida ideal pós-treino deve conter carboidrato, para repor os estoques de glicogênio muscular e sódio (SILVA et al, 2015).

Uma vez conhecidos os possíveis efeitos da desidratação em diferentes níveis, surgem perguntas do tipo: ingerir quanto, como, quando? Água pura ou soluções hidroeletrolíticas? Como avaliar o estado de hidratação? Após um período em que a recomendação de "não beber" durante o exercício predominou até 1970, surgiram protocolos de hidratação que têm como objetivo orientar os atletas ou praticantes de atividade física a se hidratarem para atingir o equilíbrio hidroeletrolítico, e os mesmos se tornaram um paradigma. Estes protocolos determinam o quanto e o que se deve ingerir e de quanto em quanto tempo os líquidos devem ser repostos durante o exercício. Diante alguns estudos, houve uma crítica a tais protocolos de hidratação seguir, com isso ressalta a ausência de dados científicos consistentes nestas orientações e defende a sede como o mecanismo fisiológico eficiente para determinar a ingestão de fluidos durante o exercício (MOREIRA et al, 2016).

A seguir (tabela 1), um resumo das principais alterações da desidratação induzida pelos exercícios, conforme resultados de diversos estudos ().

Tabela 1 - Principais alterações da desidratação presente nos exercícios

\begin{tabular}{ll}
\hline \multicolumn{1}{c}{ AUMENTO } & \multicolumn{1}{c}{ REDUÇÃO } \\
\hline $\begin{array}{l}\text { Frequência cardíaca submáxima } \\
\text { Índice de percepção de esforço }\end{array}$ & $\begin{array}{l}\text { Volume plasmático } \\
\text { Fluxo sanguíneo para pele e músculos ativos, fígado e } \\
\text { outros órgãos } \\
\text { Taxa de sudorese }\end{array}$ \\
$\begin{array}{l}\text { Náuseas e vômito } \\
\text { Temperatura interna: Hipertermia }\end{array}$ & $\begin{array}{l}\text { Pressão arterial } \\
\text { Tempo para atividade continua, prolongada e intensa }\end{array}$ \\
$\begin{array}{l}\text { Doenças do calor: cãibras, exaustão ou } \\
\text { choque térmico }\end{array}$ & Componentes cognitivos \\
\hline
\end{tabular}

Fonte: Hernandes; Nahas (2009)

\section{IMPORTÂNCIA DOS REPOSITORES DURANTE A PRÁTICA DE ATIVIDADE FÍSICA}

A hidratação contendo apenas água, embora seja indispensável, é considerável incompleta, pois, quando se refere a pessoas que realizam atividades extenuantes considera-se inadequada a reidratação contendo apenas água por provocar uma perda considerável de eletrólitos, e estar associada a um quadro de hiponatremia, como: edema cerebral, incluindo a confusão mental, letargia, anorexia, convulsões, náuseas, vômitos, cãibras e/ou fraquezas. Em atividades intensas seria interessante à reposição de bebidas esportivas de carboidrato, sódio, vitaminas e minerais acrescidos a 250 a 500 mililitros de água em temperatura por volta de 15 a $22^{\circ} \mathrm{C}$, e ainda um sabor que favoreça o gosto do indivíduo (SILVA FILHO et al, 2015).

Entre os eletrólitos, o sódio é o mais importante, pois pequena quantidade deste acrescentadas em bebidas esportivas tornam possível uma reidratação mais completa quando comparada com a água pura. Essa reposição precisa ser feita antes, durante e depois dos treinos. (LIMA et al, 2007).

Bebidas contendo concentrações razoáveis de carboidrato podem oferecer vantagens adicionais durante exercício prolongado. A sua presença auxilia na reidratação do atleta, na reposição dos estoques 
de glicogênio corporal, na prevenção da queda da glicemia após duas horas de exercício e retarda a fadiga. O carboidrato estimula a absorção de líquidos e eletrólitos no intestino delgado, aumentando mais rapidamente o volume plasmático. Quanto mais rápida a bebida for absorvida no intestino, melhor os efeitos para o atleta pois, quando incorporada é captada pelas fibras musculares ativas, ajudando a manter uma alta taxa de oxidação de carboidrato, que pode melhorar a performance e o tempo de exaustão. Para Lima et al (2015):

A maioria das bebidas esportivas possui tanto carboidrato quanto sódio, pois juntos estimulam mais rapidamente a absorção do fluido, fazendo uma rápida reidratação, diminuindo o estresse fisiológico do exercício e promovendo a recuperação após o mesmo; assim, água, carboidrato e sódio são os ingredientes chaves para a manutenção do balanço hídrico.

Esta situação, juntamente com a ingestão de bebidas hipotônicas, com baixa concentração de sódio, pode fazer com que a osmolalidade plasmática atinja valores aproximadamente de $130 \mathrm{mEq} / \mathrm{l}$. Sendo denominada hiponatremia, cujos sintomas típicos incluem náusea, vômito, cefaleia e mal-estar. Com a progressão da hiponatremia pode haver confusão, reflexos diminuídos, convulsões, estupor e coma. Em alguns esportes intermitentes como; (futebol, basquetebol, tênis e handebol), recomenda-se a ingestão concomitante de água e repositor hidroeletrolítico desde o início da atividade, visto que tais atividades promovem uma depleção acelerada das reservas de glicogênio muscular e o consumo dos repositores poderão manter os níveis glicêmicos, além de poupar o glicogênio muscular, retardando, assim, a instalação dos sintomas da fadiga (HAUSEN et al, 2013).

\subsection{Ingestão de eletrolíticos durante o exercício ou competição}

A reposição de eletrólitos e de extrema importância para o balanço osmótico das células. Recomenda-se a adição de quantidades adequadas de carboidratos e eletrólitos para eventos com duração maior do que uma hora, já que não prejudica a distribuição de água pelo organismo e melhora o desempenho de glicogênio muscular e hepático. Além disso, evita a queda que ocorre no volume do plasma: isso ajuda a manter a potência cardíaca, por meio da conservação do volume de batidas, e aumenta o fluxo do sangue para a pele, o que promove a perda de calor e limita o aumento da temperatura corporal (BANIN et al, 2010).

Segundo Drumond et al (2007), a preocupação com a ingestão de solução mais adequada nas atividades se faz necessária, pois em atividades com até 60 minutos de duração, a água é a bebida mais adequada, porém, quando as atividades são superiores a 60 minutos, a utilização de bebidas que contenham carboidrato passa a ter uma importância considerável. Ao se hidratar exclusivamente com água, os atletas apenas mantem seus estoques hídricos próximos da normalidade, mas quando fazem uso de soluções carboidratadas poderão, além de restaurar os estoques hídricos, armazenar uma quantidade extra de carboidratos para ser utilizado durante a atividade, evitar quadros de hipoglicemia e acelerar a recuperação do glicogênio muscular.

Uma bebida interessante que está sendo consumida por praticantes de atividade física é a água de coco, sendo um produto substituto aos suplementos hidroeletrolíticos, pois além da grande quantidade de potássio, possui glicose, sendo uma bebida natural, com aroma, sabor suave e que pode ser consumida por todas as idades. A água de coco é composta principalmente por açúcares, minerais, substâncias nitrogenadas, gorduras e algumas vitaminas, mas as maiores concentrações encontradas foram dos eletrólitos de potássio, de cálcio e de magnésio. A sua indicação na hidratação também é utilizada pelo 
fato de apresentar uma densidade semelhante à do plasma sanguíneo, $p H$ favorável, presença de aminoácidos essenciais, vitaminas do complexo B, ácido ascórbico e eletrólitos diversos (BOLZAN et al, 2013).

Segundo Pinto et al (2015), a água de coco vem sendo utilizada por atletas de alto desempenho como um isotônico natural devido sua alta osmolaridade, altos níveis de carboidratos, principalmente, frutose e glicose e é rica em aminoácidos, tais como lisina, cistina, fenilalanina, histidina e triptofano. Os isotônicos, ou bebidas esportivas, são bastante consumidos pela população, principalmente por atletas profissionais e por pessoas que praticam esportes. As bebidas esportivas possuem essa denominação por apresentar osmolaridade similar à do sangue humano. Todavia, a despeito de suas propriedades hidroeletrolíticas, as mesmas podem apresentar efeitos danosos sobre os dentes, os rins e a mucosa gástrica devido ao seu baixo $\mathrm{pH}$ e presença de ácido cítrico em sua composição quando consumidas de forma excessiva e/ou por períodos prolongados.

Portanto, a característica das bebidas de reposição eletrolítica deve respeitar fatores individuais, como também aqueles relacionados ao clima e à atividade desportiva. Tal reposição deve ocorrer antes, durante e após a sessão do exercício. Conforme a duração, os eventos são classificados em atividades de menos de uma hora, entre uma e três horas e acima de três horas. Em atividades com menos de uma hora de duração, a reposição de água visa a evitar o aumento da temperatura central, não sendo necessária a reposição de sódio. Nessa situação, a reposição de carboidrato não é recomendada, principalmente porque em geral são atividades de alta intensidade, nas quais o esvaziamento gástrico é prejudicado (CARVALHO; MARA, 2010).

\section{CONSIDERAÇÕES FINAIS}

De acordo com os estudos encontrados na revisão de literatura apresentada é notório que a hidratação deve ser bem orientada para que não influencie negativamente no rendimento e na saúde tanto de atletas quanto de indivíduos não-atletas. A desidratação é uma ameaça em potencial para todos os atletas, amadores e praticantes de atividade física, principalmente para aqueles que não estão aclimatados para realizar atividades extenuantes em ambientes adversos.

A ingestão de líquidos deve ocorrer antes, durante e depois da atividade física, evitando assim comprometimento da saúde do indivíduo, além do que ela se faz importante na performance e termorregulação. A necessidade de reposição de líquidos depende do volume perdido pelo suor e seu conteúdo de eletrólitos, bem como do tempo disponível até a próxima sessão de exercícios. O consumo de bebidas é de suma importância para garantir o nível de hidratação satisfatório; este procedimento é necessário para evitar que já se inicie o exercício com déficit de fluidos que poderá ser agravado com sua execução.

Portanto, alguns pontos precisam ser mais investigados, como a especificidade das demandas hídricas e eletrolíticas em diversas modalidades esportivas, tentando elucidar qual bebida responde melhor a reposição dos fluidos em diferentes contextos esportivos e momentos de hidratação.

\section{REFERÊNCIAS}

BANIN, R.M.et al. Análise do nível da perda hídrica e porcentagem da taxa de sudorese em atletas nadadores de competição da cidade de São Caetano do Sul-SP. Revista Brasileira de Nutrição Esportiva, São Paulo. v. 4. n. 19. p. 30-35. Jan/Fev. 2010. ISSN 1981-9927. 
BARROSO, S.da S.et al. Estado de hidratação e de desempenho cognitivo-motor durante uma prova de fast trianthlon no calor. Rev. Educ. Física/UEM, v. 25. n. 4. p. 639-650. 4. trim. 2014. DOI: $10.4025 /$ reveducfis.v25i4.22459.

BOLZAN, S.S.; CASTIOGLIONE, T.F.B.; ROSSI, L. Efeitos da reposição hídrica com água de coco sobre o rendimento de praticantes de Spinning. Artigo Original. O Mundo da Saúde, São Paulo, v. 37, n.3, p. 336-342. 2013.

BOURA, N.G.M. V; SILVA, R.B. Avaliação da perda hídrica em atletas de natação em uma sessão de treinamento. Revista Brasileira de Prescrição e Fisiologia do Exercício, São Paulo, v.5, n.28, p.317-321. Jul/Ago. 2011. ISSN 1981-9900.

CARVALHO, T.de; MARA, L.S.de. Hidratação e Nutrição no Esporte. Revista Brasileira de Medicina do Esporte, Florianópolis; SC, v.16, n.2, p.144-148. Mar/Abr, 2010.

CIRNE, M.R.A.; MENDES, A.C.R. Avaliação do estado de hidratação da equipe de atletismo (corrida de rua) da polícia militar da Bahia durante os treinamentos na cidade de Salvador; BA. Revista Digital, Buenos Aires. v. 16, n.161, outubro de 2011.

CRUZ, M.A.E.; CABRAL, C.A.C.; MARINS, J.C.B. Nível de conhecimento e hábitos dos atletas de mountain bike. Fitness Performance Jornaul. Rio de Janeiro, v.8. n. 2. p. 79-89. 2009. DOI: $10.3900 /$ fpj.8.2.79.p.

DRUMOND, M.G.; C., FRANCIANE R, de; GUIMARÃES, E. M.A. Hidratação em Atletas Adolescentes - Hábitos e Nível de Conhecimento. Revista Brasileira de Nutrição Esportiva, São Paulo, v.1. n.2, p. 76-93, Mar/Abr, 2007. ISSN 1981-9927.

HAUSEN, M.R; CORDEIRO, R.G; GUTTIERRES, A.P.G. Aspectos relevantes sobre a hidratação no esporte e na atividade física. Revista HUPE, Rio de Janeiro, v.12. n. 4. p. 47-58. Out/Dez, 2013. DOI: 10.12957/rhupe.2013.8712.

HERNANDEZ, A. J.; NAHAS, R.M. Modificações dietéticas, reposição hídrica, suplementos alimentares e drogas: comprovação de ação ergogênica e potenciais riscos para a saúde. Rev. Bras. Med. Esporte, v. 15. n. 3. p. 3-12. Maio/Junho. 2009.

LIMA, C.de; MICHELS, M.F.; AMORIM, R. Os diferentes tipos de substratos utilizados na hidratação do atleta para melhora do desempenho. Revista Brasileira de Nutrição Esportiva, São Paulo, v. 1, n. 1, p. 73-83, Jan/Fev, 2007. ISSN 1981-9927.

MOREIRA, C.A.M. et al. Hidratação durante o exercício: a sede é suficiente? Revista Brasileira de Medicina do Esporte, Belo Horizonte, v.12, n.6, p.405-409, Nov/Dez, 2006.

JAHNKE, J. Estratégias Ergogênicas Nutricionais de corredores amadores. Revista Brasileira de Nutrição Esportiva, São Paulo, v.5. n. 26, p. 135-144, Mar/Abr. 2011. ISSN 1981-9927.

PEREIRA, E.R.et al. Hidratação: Conceitos e formas de avaliação. Revista Científica do Departamento de Ciências Biológicas, Ambientais e da Saúde - DCBAS, Belo Horizonte, v.3, n. 2. 2010. ISSN 1984-7688.

PINTO, A.C.L.et al. Água de coco em pó como suplemento hidroeletrolítico e energético para atletas. Rev. Bras. Med. Esporte, Ceará, v.21. n. 5. p. 390-394. Set/Out. 2015. DOI: 10.1590/1517$\underline{869220152105141533 .}$ 
PINTO, S.da F.; BERDACKI, V.S.; BIESEK, S. Avaliação da Perda Hídrica e do Grau de Conhecimento em Hidratação de Atletas de Futebol Americano. Revista Brasileira de Nutrição Esportiva, São Paulo, v.8. n. 45.p. 171-179. Maio/Jun. 2014. ISSN 1981-9927.

RIBEIRO, J.P.da S.; LIBERALI, R. Hidratação e exercício físico - Revisão Sistemática. Revista Brasileira de Nutrição Esportiva, São Paulo, v.4. n.24. p.506-514. Nov/Dez. 2010. ISSN 19819927.

SILVA FILHO, J.N.da S.et al. Necessidades da Reidratação para a Saúde do Trabalhador: Uma Revisão Sistemática. Revista Brasileira de Nutrição Esportiva, São Paulo, v. 9. n. 54, p. 578586, Nov/Dez, 2015. ISSN 1981-9927.

SILVA, F. I. Cet al. A importância da hidratação hidroeletrolítica. Rev. Bras. Ciência e Movimento, Piaui. v. 19. n. 3. p. 120-128. Set/Out. 2011. ISSN 0103-1716.

SILVA, J.K.F.; LIRANI, L.da S.; RIBAS, M.R. Análise dos níveis de perda hídrica e porcentagem da taxa de sudorese em atletas adolescentes nadadores de competição no treinamento agudo aeróbico. Revista Brasileira de Nutrição Esportiva, São Paulo. v. 9. n. 52. p.326-336. Jul./Ago. 2015. ISSN 1981-9927.

WEBBER, J. et al. Alteração do peso corporal para avaliação do grau de desidratação em atletas de futsal com idade entre 18 a 32 anos de uma equipe profissional de Santa Catarina. Revista Brasileira de Nutrição Esportiva, São Paulo. v. 3. n. 18. p. 556-561. Nov/Dez. 2009. ISSN 1981-9927. 
